\title{
Elena Conti
}

\section{Macro- and micro-evolutionary perspectives on diversification: linking pattern with process}

\author{
Conti, E.: Macro- and micro-evolutionary perspectives on diversification: linking pattern with \\ process. — Bocc. 28: 249. 2019. - ISSN: 1120-4060 printed, 2280-3882 online. \\ Key words: angiosperm, evolution, phylogeny.
}

Recent reviews have addressed the state of our knowledge on angiosperm evolution, focusing primarily on macroevolutionary patterns. What is still largely missing is the link between macroevolutionary outcomes (in other words, the patterns that we can observe in the distribution of diversity across lineages) and the processes that generate these outcomes. For example, once we identify a genomic, morphological, ecological, or distributional change that appears to be correlated with a shift of diversification rates in a phylogeny, can we discover how the change affected processes of speciation and extinction? What are the likely intrinsic and extrinsic drivers that shape biological variation and species divergence in sympatry vs. allopatry? What is the relationship between species integrity and gene flow at that most challenging of hierarchical levels, where microevolution meets macroevolution? In this talk, I argue that one way of linking macro- and microevolution is by combining studies at multiple hierarchical scales in focal taxa, de facto turning them into model lineages for evolutionary biology. I will refer primarily, but not exclusively, to my work on primroses, where I try to accomplish the goal of relating macroevolutionary patterns to microevolutionary processes. The interconnections between these hierarchical levels can best be elucidated by investigating target taxa and integrating evidence from a range of methodological approaches, including phylogenetics, population genetics, comparative genomics, experimental and functional studies, and morphological and modeling analyses. I will conclude by highlighting current challenges to a more holistic understanding of evolution before suggesting possible ways forward.

Address of the author:

Elena Conti,

Deptartment of Systematic and Evolutionary Botany, University of Zurich, Switzerland. E-mail: ContiElena@systbot.uzh.ch 
